\title{
Zwischen Normalisierung und Dekonstruktion
}

\section{Queer-Heteronormativitätskritische Soziale Arbeit als Grenzbearbeitung}

Trotz einer wachsenden Sensibilität für geschlechtliche und sexuelle Vielfalt dominieren heteronormative Geschlechterbilder den gesellschaftlichen Diskurs und die Praxis der Sozialen Arbeit - zum Schaden ihrer Nutzer_innen. Um diesen entgegen zu wirken, muss sich eine queer-heteronormativitätskritische Soziale Arbeit mit den eigenen internalisierten sowie gesellschaftlich vermittelten Normalitätsvorstellungen auseinandersetzen.

$\mathrm{D}$ ie als von der Norm abweichend markierten „Anderen“ galten lange Zeit und gelten zum Teil immer noch als Zielgruppe der Sozialen Arbeit. Kessl und Plößer beschreiben dies als konstitutives Dilemma Sozialer Arbeit als „Instanz zur Bearbeitung von Differenz und Andersheit" (Kessl und Plößer 2010, S. 8). Zum einen fungiert sie als Integrationshilfe

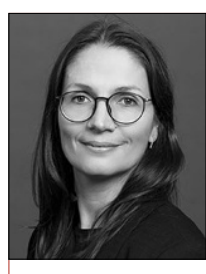

\section{Davina Höblich}

Hochschule RheinMain, Wiesbaden, Deutschland

*1977; Dr. phil., Dipl.-Pädagogin, Professorin für Soziale Arbeit mit den Schwerpunkten Bildung, Ethik und Arbeit mit Kindern und Jugendlichen an der Hochschule RheinMain.

Davina.hoeblich@hs-rm.deHannah.goede@hs-rm.de



\section{Hannah Goede}

Hochschule RheinMain,

Wiesbaden, Deutschland

*1992; M. A., wissenschaftliche Mitarbeiterin am Fachbereich

Sozialwesen der Hochschule RheinMain.

Zusammenfassung Die gesellschaftliche Matrix der Heteronormativität prägt unbewusst das professionelle Handeln von Fachkräften der Sozialen Arbeit. Dies ist kritisch zu bewerten, da Normalitätsvorstellungen als hegemoniale Grenzziehungen zum Ausschluss (vermeintlich) von der Norm abweichender Personen aus der Gesellschaft führen. Der Beitrag gibt Impulse für eine Soziale Arbeit als Grenzbearbeiterin, die sich neben der Wahrnehmung und Kritik von Grenzziehungen auch der eigenen „Denormalisierungsangst“ stellt. Im Spannungsfeld zwischen Affirmation und Dekonstruktion von Differenz werden Ansatzpunkte für eine Veränderung der hegemonialen Geschlechterordnung sichtbar.

Schlüsselwörter Heteronormativitätskritik, Geschlechternormen, Grenzbearbeitung, Soziale Arbeit als Normalisierungsmacht, Denormalisierungsangst für ihre Adressat_innen, „,in Relation zur Gesamtbevölkerung weniger ,anders" zu sein “ (Ebd.). Zum anderen übt sie „Normalisierungsmacht“ (Ebd.) aus und „passt ,die Anderen“ in diesem Sinne an die bestehenden Normen an oder produziert Nutzer_innen durch die fachliche Fallmarkierung überhaupt erst als ,Andere' (mit)“ (Ebd.). Öffentliche und politische Diskurse bestimmen, was als „soziales Problem“ oder als „Abweichung “ von der Norm gilt und mithin auch, welche Bearbeitung hier seitens der Sozialen Arbeit notwendig erscheint (Dollinger 2018, S. 70 f.). Soziale Arbeit ist hierbei aufgefordert, sich kritisch in diesen Diskursen zu positionieren, wie auch die hierin zugrundeliegenden Normalitätsvorstellungen und ihre eigene Rolle bei der (Re-) Produktion „sozialer Normen“ und ihrer kritischen Weiterentwicklung zu reflektieren. Dies erscheint umso wichtiger, als „Sozialpädagog*innen [...] mit Adressat*innen in machtförmigen, asymmetrischen Arbeitsbeziehungen “ (Ebd., S. 74) operieren, in denen die Fachkraft qua Amt über mehr Macht verfügt.

Im Folgenden setzen wir uns daher zunächst

1. mit der Bedeutung von gesellschaftlichen Normen zur Herstellung und Aufrechterhaltung von Machtverhältnissen sowie der psychischen und sozialen Funktion von Normen für Individuen auseinander,

2. betrachten anschließend die Rolle Sozialer Arbeit als Normalisierungsmacht und Grenzbearbeiterin,

3. konkretisieren diese beispielhaft am Umgang mit der gesellschaftlichen Norm der Heteronormativität und

4. loten die Chancen und Grenzen einer heteronormativitätskritischen Sozialen Arbeit als Konzept für einen reflexiven machtkritischen Umgang mit Normen am Beispiel sexueller und geschlechtlicher Vielfalt aus. 


\section{Bedeutung gesellschaftlicher Normen}

zur Herstellung und Aufrechterhaltung

\section{von Machtverhältnissen}

Soziale Normen und Werte bieten Orientierung in einer Welt der fortschreitenden Ausdifferenzierung und Pluralisierung von Lebensentwürfen. Über soziale Normen „können heterogene Wirklichkeitskonstruktionen so aufeinander bezogen werden, dass diese [...] relativ stabile Ordnungsmuster bilden und damit soziale Ordnung herstellen " (Seelmeyer und Kutscher 2018, S. 1126). Soziale Normen geben Menschen Orientierung und Handlungssicherheit (Tranow 2010, S. 346). Darüber hinaus werden über sie Gefühle der Zugehörigkeit zu einer sozialen Gruppe oder Gemeinschaft geschaffen, was sich positiv auf die persönliche Identität von Individuen auswirkt. Allerdings haben soziale Normen immer auch eine regulative und aus- bzw. abgrenzende Funktion, in dem sie festlegen, welche Verhaltensweisen und Lebensformen als „nicht-normal“ gelten und welchen Individuen dementsprechend Zugehörigkeit und Anerkennung verwehrt wird (Jäger 2014, S. 28).

Soziale Normen sind soziale Konstrukte. Sie beziehen sich nicht auf natürliche Tatsachen, sondern entstehen durch Interaktionen (Dollinger 2018, S. 70; Seelmeyer und Kutscher 2018, S. 1124). Wo die Grenze zwischen „Norm“ und „Abweichung“ verläuft, ist variabel und „eine Frage konkreter Machtverhältnisse“ (Jäger 2014, S. 28). „Normalität“ ist daher Ergebnis diskursiver Aushandlungen, in denen die Teilnahmevoraussetzungen ungleich verteilt sind (Ebd., S. 30). In der Regel sind es die „Etablierten“ (Rommelspacher 2002, S. 18), also diejenigen, die an der Spitze der sozialen Hierarchie stehen, denen die Deutungshoheit bzw. Definitionsmacht obliegt und die die Norm repräsentieren, die sie prägen.

Dadurch, dass Individuen „soziale Normen“ im Zuge ihrer Sozialisation verinnerlichen, werden diese als eigene Verhaltensstandards übernommen und durch innere Sanktionsmechanismen wie zum Beispiel Scham abgesichert (Tranow 2010, S. 343). Diese Verinnerlichung ist einer der Gründe, weshalb es so schwer ist, „Normalität“ als Modus der Herrschaftssicherung zu entlarven. Denn indem „ein grundsätzlicher (Normen-) Konsens unterstellt wird und Normen und Werte als, sozialer Kitt' propagiert werden " (Anhorn und Stehr 2018, S. 348), lassen sich Diskriminierung und soziale Ungleichbehandlung als individuelles Versagen darstellen und damit legitimieren.

\section{Soziale Arbeit als Normalisierungsmacht und Grenzbearbeiterin}

Die Veränderung von durch Normalitätskonstruktionen kaschierten Macht- und Differenzordnungen erweist sich in der Praxis (der Sozialen Arbeit) als lang- wieriger und von Uneindeutigkeiten gekennzeichneter Prozess. Diese Ambivalenzen können mithilfe des Ansatzes der Grenzbearbeitung aufgefangen werden, wodurch die transformativen Potenziale der Sozialen Arbeit nutzbar werden (Maurer 2018, S. 25). Grenzen werden hierbei ebenso wie Normen als „relationale Gebilde“ (Kessl und Maurer 2010, S. 158) verstanden, die erst in sozialen Interaktionen ihre Wirksamkeit entfalten.

Der Ansatz der Grenzbearbeitung ist weniger ein Konzept mit konkreten Handlungsanweisungen als eine Denkfigur. Diese zeichnet sich durch fortlaufende (Selbst-) Reflexionen aus, wodurch größere Handlungsspielräume und Bewegungsfreiheit erarbeitet und schließlich neue, gerechtere gesellschaftliche Verhältnisse hergestellt werden sollen (Maurer 2018, S. 22). Die implizierte Räumlichkeit des Grenzbegriffs ermöglicht es, verschiedene, sich intersektional überschneidende Differenzordnungen zu einem Zeitpunkt an einem Ort zu konkretisieren, wodurch sie analysier- und bearbeitbar werden (Ebd., S. 21 f.).

Die Grenzbearbeitung setzt an der Begrenztheit der eigenen Wahrnehmung, des eigenen Handelns und auch der Kritik an (Ebd., S. 20). Sie geht davon aus, dass die Soziale Arbeit im Sinne einer „,Ermöglichung ,Begrenzung‘ zugleich“ (Ebd., S. 25) „ihrer Normalisierungsfunktion “ (Kessl und Maurer 2010, S. 161) nicht vollends entkommen kann, da auch Sozialarbeiter_innen die etablierten sozialen Normen in ihrem Habitus verinnerlicht haben und diese unbewusst handlungsleitend wirken (Tranow 2010, S. 346). Trotz dieses Befunds sieht Maurer Möglichkeiten der Veränderung der internalisierten sowie der äußeren (Macht-) Ordnung und formuliert diese als „Momente des Vorgehens" (Maurer 2018, S. 26). Einer dieser Momente ist ihr zufolge die Sichtbarmachung der sozialen Ordnungen und ihrer hierarchisierenden Funktion sowie der eigenen Wahrnehmungsgrenzen (Ebd.).

Die Anerkennung von Diskriminierung kann jedoch auf subjektiver Ebene zu Irritationen führen, da sie in einem Widerspruch zu einem toleranten und gerechten Selbstbild steht. Das dabei entstehende Gefühl wird in der Sozialpsychologie als kognitive Dissonanz bezeichnet (Festinger 2012, S. 17). Um Konsonanz, das heißt übereinstimmende Beziehungen, Überzeugungen und Verhaltensweisen herzustellen, muss der Widerspruch zwischen Norm und Realität aufgelöst werden, etwa durch Verschleierung und Verkennung der Diskriminierung oder durch kritische, auch (fach-)politische Interventionen (Ebd., S. 16). Letztere stellen eine weitere Dimension der Grenzbearbeitung dar (Maurer 2018, S. 27).

Die tatsächliche Verschiebung bisheriger „Normalitätsgrenzen“ führt zu Verunsicherung und einer Art „Denormalisierungsangst“ (Jäger 2014, S. 28), und deshalb 
womöglich zu einem gegenüber Veränderungen abwehrenden Verhalten. Das kann sich auf die individuelle $\mathrm{Zu}-$ gehörigkeit und Identität, die Sorge um den Verlust von Deutungshoheit und Privilegien oder auf die berufliche Praxis beziehen, in der sich das Kernproblem der Kategorisierung und Grenzziehung nicht durch die Schaffung neuer Begriffe auflösen lässt (Maurer 2018, S. 24). Diese Unsicherheiten wertet der Ansatz der Grenzbearbeitung positiv als „spezifische ,Grenzerfahrungen“ in Bezug auf das bisher Gedachte und Gewollte" (Ebd., S. 26). Sie bilden die Grundlage für Transformation und Subversion, der dritten Handlungsebene der Grenzbearbeitung. Um dies zuzulassen, ist es wichtig, eine Haltung einzunehmen, in der Unsicherheit wertgeschätzt, Imagination als Werkzeug anerkannt und Konflikte und Widersprüchlichkeiten ausgehalten werden (Ebd.).

\section{Das Grenzregime Heteronormativität}

Grenzziehungen als hegemoniale Praxen der Differenzierung können sich auf tatsächliche Grenzen zwischen Staaten beziehen, auf sog. Grenzregime, wie sie in der Migrationsforschung thematisiert werden, oder auch auf die Differenzordnung der Zweigeschlechtlichkeit und Heterosexualität (Kessl und Maurer 2010, S. 159). Poststrukturalistische und queertheoretische Ansätze weisen auf die „Macht der Geschlechternormen“ (Butler 2011 [2004]) hin und darauf, dass Sexualität im Laufe der Geschichte zu einem wichtigen Schauplatz der Machtausübung und gesellschaftlichen Disziplinierung der Körper, der Bevölkerungspolitik sowie der Psychiatrisierung und Illegalisierung der „Perversionen“ (Foucault 2014 [1977], S. 135 ff.) wurde. Solche Sexualpolitiken (Rubin 1993 [1984]) grenzen Sphären der legitimen Sexualität (monogame, heterosexuelle Sexualität zwischen Cis-geschlechtlichen Personen) von als illegitim und „unnormal“ geltenden sexuellen Handlungen (Sexualität gegen Geld, One-Night-Stands, gleichgeschlechtliche Sexualität etc.) territorial ab. Menschen, die sich anders als bei der Geburt zugewiesen oder jenseits einer binären Geschlechterordnung positionieren, werden ebenso außerhalb der Grenze und damit als Abweichung von der Norm konstruiert, wie jene, deren Begehren sich nicht ausschließlich auf das "andere“ Geschlecht im Dualismus weiblich/männlich richtet.

Als implizite Annahme über Geschlecht und Begehrensformen und mithin Norm und Abweichung steuert Heteronormativität als Grenzregime wesentlich das Handeln professioneller Fachkräfte. Im Sinne von „sozialen Normen, die unsere Existenz bestimmen “ (Butler 2011 [2004], S. 9), legen normative Erwartungen fest, welche Identitätspositionen als „normal“ und legitim gelten können und welche nicht. Soziale Arbeit und die in ihr tätigen Fachkräfte agieren innerhalb von heteronormativen Gesellschaftsverhältnissen und solchen Sexualpolitiken, die sowohl seitens der Professionellen als auch der Adressat*innen internalisiert sind und von denen sie zugleich hinsichtlich der eigenen geschlechtlichen Positionierung und sexuellen Orientierung betroffen sind.

\section{Queer-heteronormativitätskritische Soziale Arbeit als Grenzbearbeitung}

Die Produktion und Konstruktion vom vermeintlichen Mainstream abweichender sexueller und geschlechtlicher Minderheiten gilt es dem Konzept der Grenzbearbeitung zufolge als soziale Ordnung mit hierarchisierender Funktion, die auch die eigene Wahrnehmung begrenzt, sichtbar zu machen (s. oben). Um eine solche Dekonstruktion vermeintlicher „Normalitäten“ im Bereich sexueller und geschlechtlicher Vielfalt vornehmen zu können und gemeinsam mit ihren Adressat_innen zu befördern, müssen sich Fachkräfte mit ihrer eigenen „Denormalisierungsangst" und darauf bezogenen eigenen Widerständen und Abwehrhaltungen kritisch-reflexiv auseinandersetzen. Hierzu gehört eine kritische Auseinandersetzung mit der eigenen sexuellen Orientierung und der eigenen geschlechtlichen Identität auch als Ausdruck von gesellschaftlichen Geschlechterregimen und der heterosexuellen Matrix. Fachkräfte stehen vor der Herausforderung, sexuelle und geschlechtliche Minderheiten und ihre gesellschaftliche Diskriminierung einerseits zu benennen und so zu einer Dekonstruktion von „Normalitäten“ beizutragen. Zugleich müssen sie die Reproduktion von "Andersheit“ und „Abweichung “ im Sinne des Otherings (Riegel 2016) und einer Sozialpädagogisierung sexueller und geschlechtlicher Minderheiten als zu Normalisierende vermeiden. In der Denkfigur der Grenzbearbeitung gilt es vielmehr sich auf „die gesellschaftlichen Verhältnisse als ,gegebene“ und machtvoll wirksame ebenso [zu] beziehen, wie auf die vielfältigen Versuche, diese in kritisch-utopischer Absicht zu problematisieren und für neue, gerechtere, solidarischere Möglichkeiten zu öffnen." (Maurer 2018, S. 22) Zu fragen ist daher, wie sich professionelle Fachkräfte mit ihren eigenen Begrenzungen und Grenzziehungen auseinandersetzen und inwieweit sie versuchen, diese in kritischer Absicht zu hinterfragen und zu bearbeiten, wie sie einen Beitrag zu Veränderungen und mithin zu Öffnungen und der Erweiterung von Lebensmöglichkeiten für sich und die Adressat_innen leisten. Diese konkreten Möglichkeiten einer verändernden Praxis und die Figur einer Sozialen Arbeit als Grenzbearbeitung entfalten dann ihre Gesellschaftskritik an der Sozialdisziplinierung wie auch ihr an Gerechtigkeit und Empowerment orientiertes emanzipatorisches (Bildungs-) Potenzial (Höblich 2020; Maurer 2018, S. 25). 
Zunächst geht es um Wahrnehmung und Markierung über Grenzen reproduzierter gesellschaftlicher Verhältnisse in Bezug auf Geschlecht und sexuelle Orientierung und mithin auch der kritischen Selbstreflexion der eigenen Verstrickung in die (Re-) Produktion derselben. Sodann zielt eine heteronormativitätskritische Soziale Arbeit auf eine „Kritik der Verhältnisse“ (Maurer 2018, S. 27) auf der Makro-Ebene gesellschaftlicher Verhältnisse (z. B. in Formen politisierender Sozialer Arbeit, Empowerment und kritischer Einmischung in Bezug auf wohlfahrtsstaatliche Politiken, Gesetzgebungen, Programme, Finanzierung), ihrer Sedimentierung z. B. über institutionalisierte Muster kultureller Wertschätzung (Fraser 2003) auf die Meso-Ebene der Institutionen (die Rolle von Geschlecht und sexueller Orientierung bei der Konstruktion von Angeboten, Adressat_innen, Arbeitsaufträgen etc.) und schließlich auf der Mikroebene der interaktiven Herstellung von Arbeitsbündnissen zwischen Professionellen und Adressat_innen (z. B. in Fragen der wechselseitigen Anerkennung und (Schutz-) Räumen für die Erprobung geschlechtlicher und sexueller Identitätsentwürfe auch jenseits des heteronormativen Mainstreams).

Im Umgang mit Normierung und Normalisierung geht es dann darum, differenzaffirmative Ansätze als Anerkennung von Differenz und Anti-Diskriminierung mit diversitätsreflexiven Ansätzen der Dekonstruktion - die Differenzen als gesellschaftlich-kulturell hervorgebrachte und daher auch veränderbare begreifen - in der eigenen professionellen Tätigkeit zu verknüpfen (Mecheril und Kourabas 2015; Höblich und Baer 2021). In diesem Spannungsfeld bzw. Schwebezustand liegt das von der Grenzbearbeitung angedeutete Potenzial, das Diskriminierungen zugrundeliegende Prinzip der Grenzziehung selbst aufzubrechen und Normen zu verlernen, da Raum für neues Denken und Handeln freigelassen wird.

Eingegangen. 1. März 2021

Angenommen. 26. März 2021

Funding. Open Access funding enabled and organized by Projekt DEAL.

Open Access. Dieser Artikel wird unter der Creative Commons Namensnennung 4.0 International Lizenz veröffentlicht, welche die Nutzung, Vervielfältigung, Bearbeitung, Verbreitung und Wiedergabe in jeglichem Medium und Format erlaubt, sofern Sie den/die ursprünglichen Autor(en) und die Quelle ordnungsgemäß nennen, einen Link zur Creative Commons Lizenz beifügen und angeben, ob Änderungen vorgenommen wurden.

Die in diesem Artikel enthaltenen Bilder und sonstiges Drittmaterial unterliegen ebenfalls der genannten Creative Commons Lizenz, sofern sich aus der Abbildungslegende nichts anderes ergibt. Sofern das betreffende Material nicht unter der genannten Creative Commons Lizenz steht und die betreffende Handlung nicht nach gesetzlichen Vorschriften erlaubt ist, ist für die oben aufgeführten Weiterverwendungen des Materials die Einwilligung des jeweiligen Rechteinhabers einzuholen.

Weitere Details zur Lizenz entnehmen Sie bitte der Lizenzinformation auf http://creativecommons.org/licenses/by/4.0/deed.de.

\section{Literatur}

Anhorn, R., \& Stehr, J. (2018). Kritische Soziale Arbeit. In G. Graßhoff, A. Renker \& W. Schröer (Hrsg.), Soziale Arbeit (S. 341-355). Wiesbaden: Springer VS.

Butler, J. (2011). Die Macht der Geschlechternormen und die Grenzen des Menschlichen. Berlin: Suhrkamp.

Dollinger, B. (2018). Abweichendes Verhalten. In G. Graßhoff, A. Renker \& W. Schröer (Hrsg.), Soziale Arbeit (S. 69-81). Wiesbaden: Springer VS.

Festinger, L. (2012). Theorie der kognitiven Dissonanz (2. Aufl.). Programmbereich Psychologie. Bern: Huber. Faksimile-Reprint der deutschsprachigen Erstausgabe von 1978

Foucault, M. (2014). Der Wille zum Wissen. Sexualität und Wahrheit, Bd. 1. Frankfurt am Main: Suhrkamp.

Fraser, N. (2003). Soziale Gerechtigkeit im Zeitalter der Identitätspolitik. Umverteilung, Anerkennung, Beteiligung. In N. Fraser \& A. Honneth (Hrsg.), Umverteilung oder Anerkennung? Eine politisch-philosophische Kontroverse (S. 13-128). Frankfurt: Suhrkamp.

Höblich, D. (2020). Soziale Arbeit als Projekt sozialer Gerechtigkeit. Dilemmata im Umgang mit Differenz am Beispiel sexuelle Orientierung. In P. Cloos, B. Lochner \& H. Schoneville (Hrsg.), Soziale Arbeit als Projekt. Konturierung von Disziplin und Profession (S. 119-129). Wiesbaden: Springer VS.

Höblich, D., \& Baer, S. (2021). Umgang mit sexueller und geschlechtlicher Vielfalt - Herausforderungen affirmativer Praxen. Sozial Extra. https:// doi.org/10.1007/s12054-021-00364-0.

Jäger, M. (2014). Rassismus und Normalität im Alltagsdiskurs. Anmerkungen zu einem paradoxen Verhältnis. In A. Broden \& P. Mecheril (Hrsg.), Rassismus bildet. Bildungswissenschaftliche Beiträge zu Normalisierung und Subjektivierung in der Migrationsgesellschaft. Soziologische Theorie. (S. 27-39). Bielefeld: transcript.

Kessl, F., \& Maurer, S. (2010). Praktiken der Differenzierung als Praktiken der Grenzbearbeitung. Überlegungen zur Bestimmung Sozialer Arbeit als Grenzbearbeiterin. In F. Kessl \& M. Plößer (Hrsg.), Differenzierung, Normalisierung, Andersheit. Soziale Arbeit als Arbeit mit den Anderen (S. 154-169). Wiesbaden: VS.

Kessl, F., \& Plößer, M. (2010). Differenzierung, Normalisierung, Andersheit. Soziale Arbeit als Arbeit mit den Anderen - eine Einleitung. In F. Kessl \& M. Plößer (Hrsg.), Differenzierung, Normalisierung, Andersheit. Soziale Arbeit als Arbeit mit den Anderen (S. 7-16). Wiesbaden: VS.

Maurer, S. (2018). Grenzbearbeitung. Zum analytischen, methodologischen und kritischen Potenzial einer Denkfigur. In B. Bütow, J.-L. Patry \& H. Astleitner (Hrsg.), Grenzanalysen. Erziehungswissenschaftliche Perspektiven zu einer aktuellen Denkfigur (S. 20-33). Weinheim, Basel: Beltz Juventa.

Mecheril, P., \& Kourabas, V. (2015). Von differenzaffirmativer zu diversitätsreflexiver Sozialer Arbeit. Sozialmagazin, 2015(9/10), 22-29.

Riegel, C. (2016). Bildung - Intersektionalität - Othering. Pädagogisches Handeln in widersprüchlichen Verbältnissen. Pädagogik. Bielefeld: transcript. https://doi.org/10.14361/9783839434581.

Rommelspacher, B. (2002). Anerkennung und Ausgrenzung. Deutschland als multikulturelle Gesellschaft. Frankfurt am Main, New York: Campus.

Rubin, G. (1993). Thinking sex. Notes for a radical theory of the politics of sexuality. In H. Abelove (Hrsg.), The lesbian and gay studies reader (2. Aufl. S. 143-178). New York: Routledge.

Seelmeyer, U., \& Kutscher, N. (2018). Normalität und Normalisierung. In H.-U. Otto, H. Thiersch, R. Treptow \& H. Ziegler (Hrsg.), Handbuch Soziale Arbeit. Grundlagen der Sozialarbeit und Sozialpädagogik (6. Aufl. S. 1124-1131). München: Ernst Reinhardt.

Tranow, U. (2010). Norm, soziale. In J. Kopp \& B. Schäfers (Hrsg.), Grundbegriffe der Soziologie (10. Aufl. S. 343-346). Wiesbaden: VS. 